\title{
JWB82.pdf
}

\section{Slow light bullets in arrays of nonlinear Bragg-grating waveguides}

\author{
Andrey A. Sukhorukov and Yuri S. Kivshar \\ Nonlinear Physics Centre and Centre for Ultrahigh-bandwidth Devices for Optical Systems (CUDOS), \\ Research School of Physical Sciences and Engineering, \\ Australian National University, ACT 0200 Canberra, Australia \\ Home page: http://www.rsphysse.anu.edu.au/nonlinear
}

We show that in specially designed nonlinear waveguides with phase-shifted Bragg gratings it is possible to realize the frequency-independent spatial diffraction in the vicinity of band-gap, allowing for efficient spatio-temporal self-trapping of slow-light bullets.

\section{(c) 2005 Optical Society of America} OCIS codes: $190.5940 ; 050.2770$

Nonlinear response of optical materials is the fundamental phenomenon leading to interactions of optical waves which enable all-optical switching for various applications. Nonlinear self-action may allow for simultaneous spatial and temporal self-trapping of optical pulses in the form of light bullets (see Ref. ${ }^{1}$, and references therein). The efficiency of nonlinear interactions is greatly enhanced in the regime of light propagation with a small group velocity. Such slow-light regime can be realized in periodic structures such as Bragg gratings ${ }^{2}$ or photonic crystals (see Ref. ${ }^{3}$, and references therein), when the frequency is tuned close to the edge of a photonic band-gap. However, for the commonly considered $2 \mathrm{D}$ or $3 \mathrm{D}$ defect-free photonic crystal structures, the spatial diffraction of light is strongly frequency-dependent in the vicinity of a band-edge. Diffraction usually increases for smaller group velocity due to the shrinkage of equal-frequency contours, and this inherently limits the efficiency of spatial self-focusing for slow-light components. Indeed, only quasi-localized nonlinear states with large spatial extent were predicted for a slab 2D waveguide with a $1 \mathrm{D}$ Bragg grating ${ }^{4}$. In this work, we show that this restriction can be overcome in specially designed arrays of Bragg-grating waveguides, where it is possible to realize the frequency-independent diffraction in the vicinity of the gap edge, allowing for the formation of strongly localized slow-light bullets.

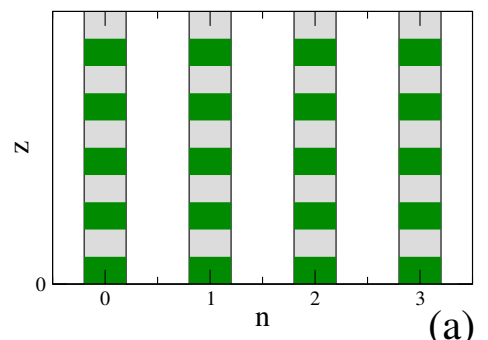

(a)

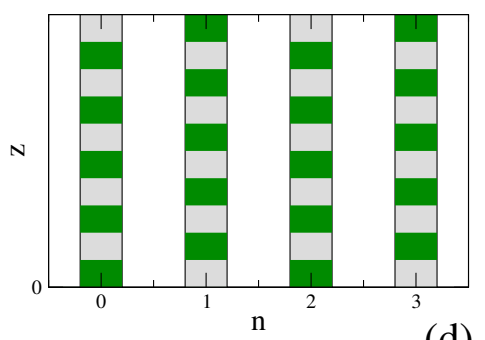

(d)

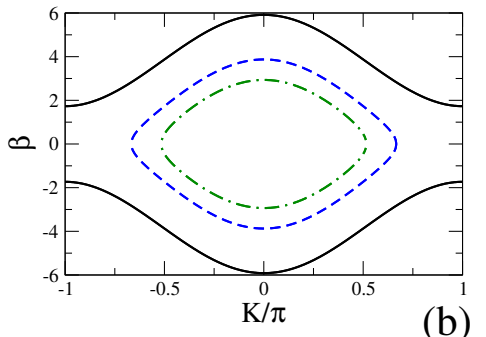

(b)

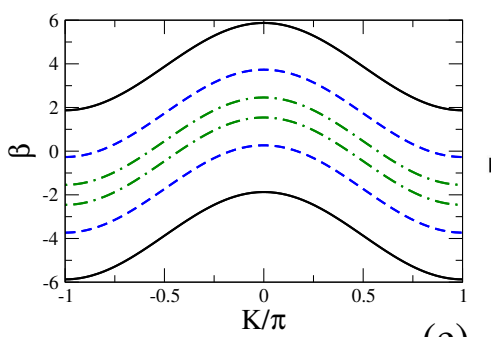

(e)
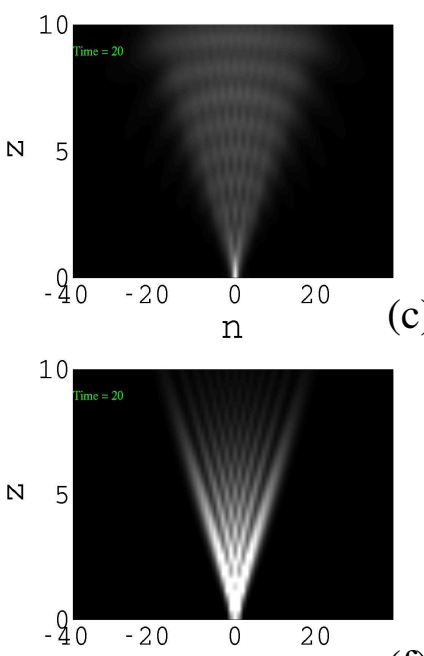

(f)

Fig. 1. (a,d) Schematic of waveguide arrays with (a) in-phase $(\varphi=0)$ and (d) phase-shifted $(\varphi=\pi)$ Bragg gratings; (b,e) Corresponding equal-frequency contours for different detunings from the gap edge: $\omega=4$ (solid), $\omega=2$ (dashed), $\omega=1.1$ (dashed-dotted line). (c,f) Corresponding diffraction patterns for a pulse with the central frequency $\omega=1$.

The arrays of nonlinear optical waveguides which are homogeneous in the propagation direction have been extensively investigated in recent years, and many possibilities for spatial beam control were demonstrated experimentally ${ }^{5,6}$. We suggest that new regimes of spatio-temporal dynamics can be accessed in waveguide arrays which are modulated in the propagation direction with the period satisfying the Bragg-resonance condition at the operating frequency. Such structures may be fabricated in AlGaAs samples, with accessible fast Kerr-type optical nonlinearity.

In the vicinity of the Bragg resonance, the evolution of optical pulses can be modeled by a set of coupled-mode equations for the slowly varying envelopes of forward $\left(u_{n}\right)$ and backward $\left(v_{n}\right)$ propagating modes in each of the waveguides $(n)$. In the normalized form, the equations can be written as $i \partial u_{n} / \partial t+i \partial u_{n} / \partial z+C\left(u_{n-1}+u_{n+1}\right)+$ $\rho_{n} v_{n}+\gamma\left(\left|u_{n}\right|^{2}+2\left|v_{n}\right|^{2}\right) u_{n}=0, i \partial v_{n} / \partial t-i \partial v_{n} / \partial z+C\left(v_{n-1}+v_{n+1}\right)+\rho_{n}^{*} u_{n}+\gamma\left(\left|v_{n}\right|^{2}+2\left|u_{n}\right|^{2}\right) v_{n}=0$, where $t$ 


\section{JWB82.pdf}

and $z$ are the dimensionless time and propagation distance, respectively, $C$ is the coupling coefficient for modes in the neighboring waveguides, $\rho_{n}$ characterize the efficiency of scattering from the Bragg-grating, $\gamma$ is the nonlinear coefficient, and the group velocity away from the Bragg resonance is normalized to 1.

We find that diffraction and dispersion of waves can be precisely tailored by introducing a phase shift between the otherwise equivalent waveguide gratings, as illustrated in Figs. 1(a,d). Only two- and three-waveguide couplers with in-phase gratings were analyzed before ${ }^{7,8}$. Here, we consider the effect of linear phase shift of gratings across the array characterized by the scattering coefficients $\rho_{n}=\rho_{0} \exp (-i \varphi n)$. Then, the Bloch eigenmodes have the form $u_{n}=u_{0} \exp ^{i K n+i \beta z-i \omega t}$ and $v_{n}=v_{0} \exp ^{i(K+\varphi) n+i \beta z-i \omega t}$, where $K$ and $\beta$ are the transverse and longitudinal components of the Bloch wave-vector. The dispersion relation follows from the eigenmode equations, $u_{0}[\omega-\beta+$ $2 C \cos (K)]+v_{0} \rho=0$ and $u_{0} \rho+v_{0}[\omega+\beta+2 C \cos (K+\varphi)]=0$. For in-phase gratings $(\varphi=0)$, the dispersion relation is $\omega(K, \beta)=-2 C \cos (K) \pm\left(\rho^{2}+\beta^{2}\right)^{1 / 2}$, and wave diffraction characterized by the curvature of the equal-frequency contours $\left(\partial \beta^{2} / \partial K^{2}\right)$ is strongly frequency-dependent, see Fig. 1(b). For out-of-phase shift of neighboring gratings $(\varphi=\pi)$, the dispersion relation is different, $\omega(K, \beta)= \pm\left\{\rho^{2}+[\beta-2 C \cos (K)]^{2}\right\}^{1 / 2}$. Most remarkably, in this case the shape of equal-frequency contours does not depend on frequency, see Fig. 1(e), and diffraction pattern of a narrow pulse [Fig. 1(f)] is essentially the same as for CW beams ${ }^{5,6}$. Moreover, for such a structure the dispersion and diffraction can be controlled independently since the frequency gap width and position does not depend on the propagation angle, i.e. there always exists a 2D (quasi-)gap. This is different from the case of $\varphi=0$, where the $2 \mathrm{D}$ gap exists only when the waveguide coupling is weak, $C<\rho / 2$. We note that the $2 \mathrm{D}$ gap appears for the modes localized in the high-index waveguides, i.e. below the light-line of substrate waves, and the maximum group index should be limited only by scattering losses, similar to the case of photonic crystal membranes exhibiting out-of-plane losses ${ }^{3}$.

We perform numerical simulations of the spatio-temporal pulse dynamics. In the linear regime, the pulse broadens both in transverse and longitudinal directions [Figs. 2(a-d)]. Nonlinear self-action results in self-trapping in space and time [Figs. 2(e-h)]. In this example, the velocity of light bullet is below $30 \%$ of the speed of light in the absence of the Bragg grating, and smaller velocities can be accessed as well by controlling the frequency of the input pulse.

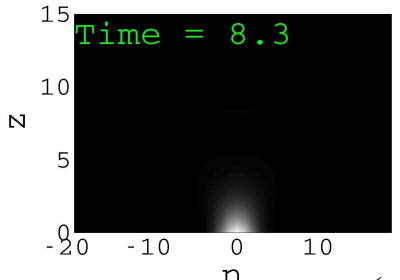

(a)

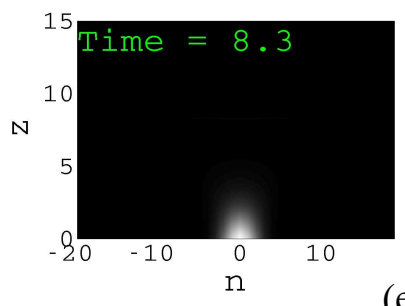

(e)

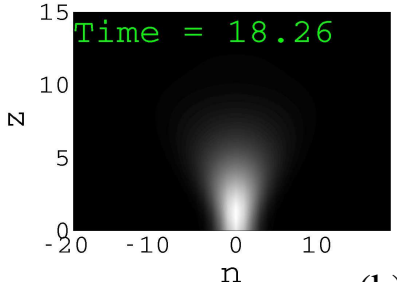

(b)

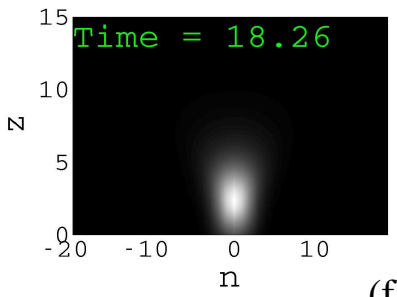

(f)

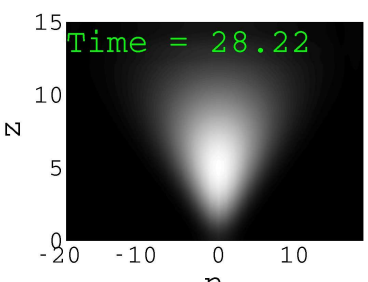

(c)

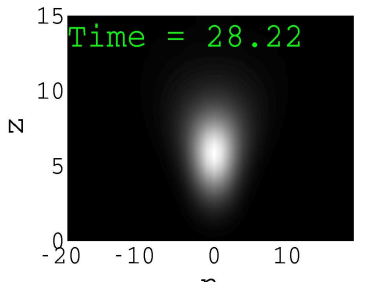

(g)

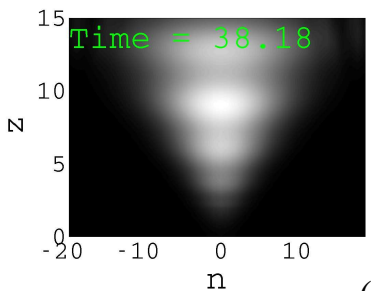

(d)

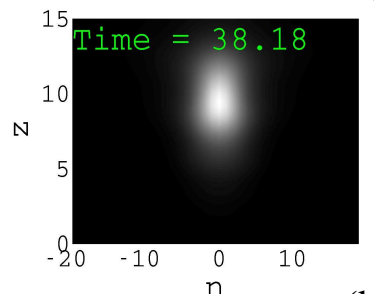

(h)

Fig. 2. Snapshots of field intensities for an optical pulse propagating in a waveguide array structure show in Fig. 1(d): (a-d) Linear broadening due to spatial diffraction and temporal dispersion; (e-h) Nonlinear self-trapping in space and time and formation of an optical bullet.

In conclusion, we have demonstrated that novel regimes of spatio-temporal dynamics can be realized in waveguide arrays with phase-shifted Bragg gratings, providing optimal conditions for the formation of slow optical bullets. These nonlinear states may offer new possibilities for all-optical switching and control of light.

\section{References}

1. H. S. Eisenberg, R. Morandotti, Y. Silberberg, S. Bar Ad, D. Ross, and J. S. Aitchison, Phys. Rev. Lett. 87, 043902 (2001).

2. B. J. Eggleton, C. M. de Sterke, and R. E. Slusher, J. Opt. Soc. Am. B 16, 587 (1999).

3. Y. A. Vlasov, M. O'Boyle, H. F. Hamann, and S. J. McNab, Nature 438, 65 (2005).

4. T. Dohnal and A. B. Aceves, Stud. Appl. Math. 115, 209 (2005).

5. D. N. Christodoulides, F. Lederer, and Y. Silberberg, Nature 424, 817 (2003).

6. J. W. Fleischer, G. Bartal, O. Cohen, T. Schwartz, O. Manela, B. Freedman, M. Segev, H. Buljan, and N. K. Efremidis, Opt. Express 13, 1780 (2005).

7. W. C. K. Mak, B. A. Malomed, and P. L. Chu, Phys. Rev. E 69, 066610 (2004).

8. A. Gubeskys and B. A. Malomed, Eur. Phys. J. D 28, 283 (2004). 